that the vast resources of teaching and experience at our large country hospitals are to a great extent wasted as means of clinical teaching. It is notorious that clinical teaching in London is not equal to the demand. Why, then, should not an effort be made to utilise the large amount of clinical experience that lies dormant in our provincial hospitals ? Supposing, for instance, that at the South Devon Hospital the physicians and surgeons were to give each of them once a week a clinical lecture on cases under their care in the hospital ; and supposing that their visits to the hospital were paid on regular days and at regular hours, with the express purpose of imparting knowledge to students, is it not possible that a vast amount of good might accrue to every one concerned? The hospital would be visited more regularly and systematically, the physicians and surgeons would undoubtedly be stimulated to a more exact and careful study of disease, and students would have a wider and more varied field for study and observation. I have strong reason to believe that if such a plan were adopted, the examining bodies in London would recognise it as part and parcel of the curriculum of medical education.

Much as I desire to avoid the question, and certain as I am that you are all heartily sick of it, I feel however compelled to call your attention to the thread-bare subject of the Contagions Diseases Acts. I do so because we have entered on a new phase.

The administration of the Acts at the Royal Albert Hospital was all that could be desired. The results were twofold ; first, an undoubted diminution of venereal disease; and secondly, a very eonsiderable gain to the morality of the three towns. I am one of those who constitute, I am sure, the great majority of our profession, to whom the idea of curing venereal disease for the more economic consideration of profit or loss to the two services is utterly repulsive. As one bound by professional duty to use my best endeavours to cure disease, and to prevent it, I treat venereal disease as I would treat small-pox or scarlet fever. But that is a matter quite distinct from the action either of the State or a community, and I hold that for any public body to set themselves to cure venereal disease without at the same time making the very best provision within their power to abate the moral evil, and to afford adequate means of reformation to the anfortunate women who are the victims of the disease, deserves strong condemnation. Hitherto this has not been the case, and it is within my own personal knowledge that the reformatory work which was accomplished at the Royal Albert Hospital, to a great extent due to the tact and zeal of the late matron Miss Farro, was of a most satisfactory character. It is aseless to refer to the action of those theoretical philanthropists who, ignorant of the practical working of the Acts, have succeeded in bringing all this work to an end. It is, however, a most extraordinary fact that, in this place where the results of the Acts are well known, men in all positions of life, of all creeds and of every shade of politics, agree in regretting their abolition. Neither would it be profitable to investigate the psychological condition of a House of Commons that could sanction their repeal in the face of the report of a Royal Commission in their favour. We may leave such a consideration to our worthy secretary, whose knowledge of lunacy enables him better t) deal with such a question. But the fact remains that the repea! of the Acts has been attended with a considerable increase of disease and immorality in the three towns.

With regard to the increase of venereal disease, I will not trouble you with any long statistics, but merely place before you the returns of the naval and military hospitals. From the former I have received returns for the years 1880 to 1886 inclusive. In the year 1880 the percentage of admissions into the naval hospital of cases of primary syphilis was 9.45 . In 1886 the percentage was 18.66 .

The military returns are infinitely more striking. I have here the returns from 1878 to 1887 . In 1878 the ratio per 1,000 admitted into hospital for primary syphilis was 30 ; in 1887 it has risen to 209 per 1,000. These figures speak for themselves as to the increase of disease. With regard to the increase of immorality, there is a very general consensus of opinion that juvenile prostitution has much in. creased. Under the deterrent powers of the Acts young girls were almost entirely prevented from following the avocation of a prostitute, and through the intervention of the police many children on the brink of ruin were rescued, and sent either to reformatories or restored to their parents. Now prostitutes of the ripe age of 10 or 12 roam our streets unmolested, and I am assured by nersons engaged in rescue. work that it is not unusual to find children of that age affected with venereal disease. When at last the Government grant was entirely withdrawn from the Royal Albert Hospital, and the lock wards wero closed, those who were engaged in reformatory work were met with this difficulty, that in the event of a woman who desired to be admitted int a home being found, as is frecuently the case, diseased, there was no place to which she could be sent for cure. Thus reformatory work was much impeded-in fact, nearly brought to an end.

This difficulty has been, in a measure, overcome. The Government have made a grant to the Royal Albert Hospital which has enabled them to re-open thirty beds for the treatment of venereal disease. So far as it may meet the difficulty of those engaged in reformatory work, we (for I think I speak here the mind of many gentlemen in Ply mouth with whom I am associated in this matter) approve of the movement; but we have yet to know whether it will be of much assistance. Beyond that I fear little benefit will be obtained, for this reason, that there is no compulsory power to retain a woman in hospital until she is cured, and that, as a matter of fact, women leave the hospital uncured ; neither is there any power of periodical examination, the result being that no woman will apply for admission to the hospital until the disease is so far advanced as to prevent her from further plying her trade. That the Government should pay for the cure of women under such circumstances, seems to me and to others to be a waste of money, also to be indirectly an encouragement to prostitution, without even the pretext of providing immunity from disease. To be in the least degree effective, there must be some power of detention in hospital until the women are proniounced free from disease, and for this power at least I think we should earnestly strive.

I trust, moreover, that the same wisdom which, in times past, has guided the executive of the Royal Albert Hospital, will still be exerted to find some method by which the work may be more satisfactorily carried out, both morally and physically. We, as members of an influential society extending throughout the United Kingdom, can, in this matter, as in others, do much to correct errors and to form a healthy public opinion; and I still live in hopes that the com. mon-sense of the nation will, before long, in some shape or other reimpose those Acts which we are convinced were on all grounds so beneficial to the community.

\section{NOTE ON THE CHEMISTRY OF STROPHANTHIN.}

\section{BY THOMAS R. FRASER, M.D., F.R.S.}

Professor of Materia Medica in the University of Edinluurgh.

SiNCE my former communications, in which several facts relating to the chemistry of strophanthus hispidus have been stated, I have completed a systematic examination of various parts of this plant, and more particularly of the seeds. Reserving a detailed description of the results of this examination, I propose now to mention only a few of these results in a brief form.

The active principle to which I have given the name "strophanthin," occurs most abundantly in the seeds. By a very simple process, consisting essentially of the separation of oil by means of ether from the alcoholic extract, I obtained some years ago a crystalline body, having great pharmacological activity, and possessing the characteristics of : a glucoside. In subsequent experiments, however, although the same process was followed, a well-marked crystalline product was not always obtained, and it soon became ovident that this difference was due to some difference in the condition of the seeds which had been operated with. Thus, from seeds collected by the late Bishop Mackenzie about 1868 , and also from seeds sent to me by Mr. Buchanan, of Blantyre, East Africa, in 1881, I had no difficulty in separating an active principle in the form of well-marked minute crystals ; but from seeds obtained from $\mathrm{Mr}$. Buchanan in 1885 , and also from seeds liberally placed at my disposal by Mr. Moir and by Messrs. Burroughs and Wellcome last year, I tailed to obtain an equally definite crystalline body. I also found that the body obtained by the process formerly described, whether in well-defined crystals or not, was resolvable by acetate of lead into at least two bodies, one of which is an extremely active glucoside, and the other an acid, for which I would suggest the name "kombic acid." It having become apparent, therefore, that the strophanthin first described is not a simple substance, attempts were made to improve the process, so as to separate strophanthin in a more pure form than I had originally succeeded in doing. The result of these attempts has been the adoption of a process whose essential steps are the following :-

Starting with the product obtained by the earlier process, it is dissolved in water, tannic acid is added to the solution, the tannate is digested with recently precipitated oxide of lead, and then extracted with rectified and proof spirit. This extract is dissolved in a small quantity of rectified spirit, and the solution is precipitated by ether. 'The precipitate is finally dissolved in weak alcohol, and through this solution carbonic acid is passed for several hours, by which means 
lead is completely got rid of. After filtration, the solution is ovaporated at a low temperature, and the product is dried in vacuo over sulphuric acid. In the process of drying it first assumes a translucent, gummy appearance, and then becomes opaque and white.

Strophanthin thus obtained is imperfectly crystallised, neutral or very faintly acid in reaction, intensely bitter, freely soluble in water, less so in rectified spirit, and practically insoluble in ether and chloroform. It burns without residue, and it does not contain nitrogen. When subjected to ultimate analysis, its percentage composition, tak ing, for the sake of brevity, the average of several closely agreeing combustions, was found to be: Carbon, 55.976; hydrogen, 7.754 oxygen, 36.283. This percentage composition fairly corresponds with the formula, $\mathrm{C}_{20}, \mathrm{H}_{34}, \mathrm{O}_{10}$.

The effects of a large number of reagents upon it have been determined. In the meantime, the following may be stated. Strong sulphuric acid produces a bright green colour, which soon becomes greenish-yellow and brown; sulphuric acid and bichromate of potash, in addition to the changes produced by sulphuric acid, a blue colour ; phosphomolybdic acid, after contact for a few hours, a bluishgreen, which, on the addition of a few drops of water, becomes pure blue; nitric or hydrochioric acid, a pale yellowish-brown ; and caustic potash, ammonia, and other alkalies a faint yellow. With a 1 per cent. solution in water, phosphomolybdic acid causes, somewhat slowly, a bright green colour, which after prolonged contact becomes greenish-blue; nitrate of silver, a reddish-brown colour and a slight dark precipitate ; caustic potash and other alkalies, a very faint yellow ; dilute sulphuric acid a faint white opalescence ; and tannic acid, an abundant white precipitate, soluble in excess both of strophanthin and of tannic acid. The solution, tested at the ordinary tomperature, is not changed in appearance by acetate or subacetate of lead, perchloride of platinum, chloride of gold, perchloride of iron, perchloride of iron and sulphuric acid, perchloride of mercury, sulphate of copper, bichromate of potassium, iodide of potassium, nor by many other reagents; except that nearly all acid reagents cause the solution to become slightly hazy, and it is then found that the solution contains glucose. This decomposition is also produced by sulphuretted hydrogen, more distinctly and rapidly after gentle heating, and for this reason it is notadvisable to use sulphuretted hydrogen in any process for preparing strophanthin.

Indeed, all the mineral acids, excepting carbonic acid, and many of the organic acids, resolve strophanthin, even in the cold, into glucose and a substance which I have named "strophanthidin." A very pretty crystallisation of the latter may be obtained in a few hours when strophanthin is dissolved in a 1.5 per cent. solution of sulphuric acid. Contact at the ordinary temperature for even three days with dilute sulphuric acid does not apparently entirely decompose the strophanthin, as an additional quantity of glucose seems to be afterwards produced when the solution filtered from strophanthidin is heated at $212^{\circ} \mathrm{F}$. for a few hours. Thus. when strophanthin was decomposed at the ordinary temperature by contact for about three days with 1.5 per cent. sulphuric acid, there was obtained 37.5 per cent. of crystalline strophanthidin, and, by estimation with Fehling's solution, about 20 per cent. of glucose. ${ }^{1}$ The crystals of strophanthidin having been removed by filtration, and the almost colourless, bitter, and acid fluid having been boiled for four hours, it was now found that the glucose had increased to 26.64 per cent., and that about 4.3 per cent. of an amorphous, brownish substance had been formed.

This action of acids renders it apparent that an acid, and especially a mineral acid, should not be used in the preparation of strophanthin. Thus, in 1877, seven years after the publication of my first communications on this subject, Hardy and Gallois described a process in which, by using for the extraction of the seeds rectified spirit acidulated with hydrochloric acid, they obtained a crystalline product which they believed to be strophanthin. There can be little doubt, however, that their crystalline product was partially, if not entirely, strophan. thidin, not only because the process they employed would decompose the strophanthin into strophanthidin and glucose, but also because their crystaliine product was found by them not to yield glucose when it was heated with dilute sulphuric acid. Hence they concluded that strophanthin is not a glucoside (Comptes Rendus, lxxxiv, 1877, p. 261; and Journal de Pharm. et de Chim., xxv, 1877, p. 177). The glucosidal character of strophanthin has now been amply demonstrated by a large number of experiments which I have made, and by the experiments of subsequent observers, and especially by those of Mr. A. W. Gerrard, described in an interesting paper published this year (the

1 In the solution obtained by this decomposition the exact estimation of glucose by means of Fehling's solution is rendered difficult and uncertain by a green hue being produced and persisting after the blue colour of Fehling's solution has disappoarod.
Pharmaceutical Journal and Transactions, May 14th, 1887, p. 923). Further, the solution obtained when strophanthin is decomposed at $212^{\circ} \mathrm{F}$. by dilute sulphuric acid has been fermented with yeast, and carbonic acid, representing 23.64 per cent. of the strophanthin, has been obtained.

\section{THE TREATMIENT OF STRICTURE OF THE URETHRA BY ELECTROLYSIS.}

(Bring an answer by Dr. Robert Newman, of New York, to THE OBJECTIONS RAISED TO THE TREATMENT.)

Communicated by W. E. STEaVenson, M.D.Cantab., M.R.C.P., In charge of the Electrical Department at St. Bartholomew's Hospital.

Dr. Ropert Newman, "of New York, has recently published his second 100 cases of stricture of the urethra treated by electrolysis, in a paper he read before the American Medical Association at Chicago. The paper has not yet been printed, but Dr. Newman has kindly sent me an abstract with answers to the numerous criticisms on and objections to this mode of treatment of stricture. I have from time to time been in communication with Dr. Newman, and have acquainted him with the questions and objections which have been raised when any reference has been made to the first hundred successful cases published by him in 1885. I will here take the opportunity of giving his own answers.

In the second hundred cases just published, "strictures which admitted no instrument were enlarged respectively from No. 17 to No. 28 (French); other strictures, admitting instruments from No. 2 and upwards, even to No. 25, were enlarged by electrolysis gradually to No. 17 and No. 32 (French) respectively.

"The duration of the strictures at the time the patients presented themselves for treatment varied from one month to thirty years.

"In the first series of 100 cases we find 42 single, and 58 muitiple, strictures, with a total of 189 strictures. In the second series of 100 cases, we have only 21 single, 79 multiple, with a total of 230 strictures.

"In the 200 cases there was 1 stricture in $31 \frac{1}{2}$ por cent.

$$
\begin{aligned}
& \text { " were } 2 \text { strictures in } 38 \frac{1}{2} \text { " }
\end{aligned}
$$

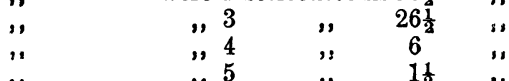

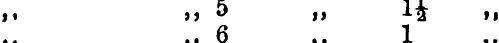

"The location of the strictures"was found"in all parts of the urethra as follows :-

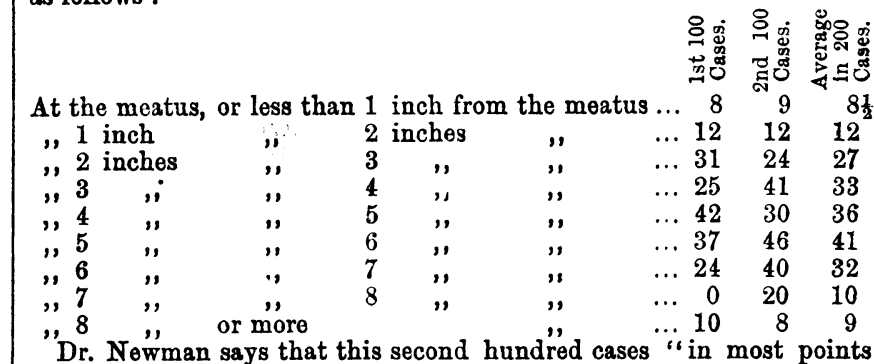
Dr. Newman says that this second hundred cases "in most points
confirms the good results of the method of treating strictures by electrolysis. The selection of the second series of 100 cases differs somewhat from the first hundred cases reported formerly, in which the salient point was to ascertain whether a relapse had taken place in a reasonable time after the patient had been discharged.

"This second hundred cases were consecutive, taken from the notebook, embracing all such patients who had remained under treatment long enough to expect a result, and who had given a complete record in order to use it for the tabular statistics."

With regard to the objections that have been raised to this mode of treatment by electrolysis, Dr. Newman writes :-

"In reelity there can be no valid objections to the method of electrolysis in the treatment of urethral strictures, and those which have been raised from time to time come either from men entirely ignorant of the first physical laws of electricity, or from those who have a personal interest or feeling in the matter. To the latter class belong some surgeons of high standing, who are wedded to the knife, have not tested the electrolysis, and hence are opposed to any innovations. Most of the objections are entirely unfounded, based on false theories, or are too trivial and even ludien as to be considered. Some have 